\title{
AN EXAMPLE OF RANK TWO SYMMETRIC OSSERMAN SPACE
}

\author{
ZORAN RAKIĆ
}

\begin{abstract}
Recently, Blažić, Bokan and Rakić, obtained some classes of 4-dimensional Osserman pseudo-Riemannian manifolds. One of these is the class of rank 2 locally symmetric space endowed with an integrable para-quaternionic structure. In this paper we give an explicit construction of an example of a space of that kind.
\end{abstract}

\section{INTRODUCTION}

Let $(M, g)$ be a 4 -dimensional pseudo-Riemannian manifold of signature $(2,2)$. Let $S_{p}^{-}$(respectively, $S_{p}^{+}$) be the set of all unit timelike (spacelike) vectors in the tangent space $T_{p} M$. The curvature or Jacobi operator $R_{X}: Y \longmapsto R(Y, X) X$ is a symmetric endomorphism of $T_{p} M$ which restricts to the endomorphism $\mathcal{K}_{X}$ of the orthogonal complement, $T_{X} S_{p}^{\varepsilon}$, of $X \in S_{p}^{\varepsilon}$ ( where $\varepsilon= \pm$ ).

DEFINITION 0.1: $M$ is timelike (respectively, spacelike) Osserman if the Jordan form of $\mathcal{K}_{X}$ is independent of $X \in S_{p}^{-}$(respectively, $X \in S_{p}^{+}$) and of $p \in M$.

For Riemannian manifolds, Osserman [5] made the following conjecture:

Conjecture (Osserman). If the eigenvalues of the Jacobi operator $\mathcal{K}_{X}$ are independent of the choice of unit vectors $X \in T_{p} M$ and of the choice $p \in M$, then either $M$ is locally a rank-one symmetric space or $M$ is flat.

Chi [2] proved the conjecture for $n \neq 4 k, k>1$. He has obtained some related results $[\mathbf{3}]$. The Osserman conjecture and related topics were studied by Gilkey, Swann and Vanhecke [4].

Definition 0.1 is the natural generalisation of the Osserman condition in the pseudoRiemannian case. If $M$ is 4-dimensional pseudo-Riemannian manifold of signature $(2,2)$, the Osserman condition is equivalent to the independency of the minimal polynomial of the Jacobi operator $\mathcal{K}_{X}$ of $X$ and $p \in M$.

Received 27th March, 1997

Research partially supported by Science Foundation of Serbia, project \#0401.

I would like to express my gratitude to Professor O. Kowalski and especially Professor D. Alekseevsky for useful conversation and suggestions about this problem. Also, I would like to express my gratitude to N. Blažić and N. Bokan who introduced me to this topic and encouraged me in the research through last year.

Copyright Clearance Centre, Inc. Serial-fee code: $0004-9729 / 98 \quad \$ A 2.00+0.00$. 
The main result of this paper is the explicit construction of an example of a timelike Osserman rank two symmetric space which is given in the proof of Theorem 1.1. This example shows the difference between of the Osserman manifolds in Riemannian and pseudo-Riemannian geometry. In the paper [1] we proved the following theorem, on which we base the present construction.

TheOREM 0.2 . (i) There exists a symmetric pseudo-Riemannian space $M$ with a metric of signature $(2,2)$ such that the matrix $\mathcal{K}_{E_{1}}$ of its Jacobi operator in the orthonormal basis, $E_{V}=\left\{E_{1}, E_{2}, E_{3}, E_{4}\right\}$, where $E_{1}$ and $E_{2}$ are timelike vectors, and $E_{3}$ and $E_{4}$ are spacelike vectors, is :

$$
\mathcal{K}_{E_{1}}=\left[\begin{array}{ccc}
-\frac{1}{2} & \frac{1}{2} & 0 \\
-\frac{1}{2} & \frac{1}{2} & 0 \\
0 & 0 & 0
\end{array}\right]
$$

(ii) The nonzero components of the curvature tensor $R$ in the basis $E_{V}$ are :

$$
\begin{aligned}
\frac{1}{2} & =R_{1221}=R_{4334}=R_{1331}=R_{4224}=R_{1224}=R_{1334}=R_{1342}, \\
-\frac{1}{2} & =R_{2113}=R_{2443}=R_{1234},
\end{aligned}
$$

(iii) The holonomy algebra $\mathfrak{h}$ of $M$ is 1-dimensional generated by:

$$
m=\left[\begin{array}{cccc}
0 & -1 / 2 & 1 / 2 & 0 \\
1 / 2 & 0 & 0 & 1 / 2 \\
1 / 2 & 0 & 0 & 1 / 2 \\
0 & 1 / 2 & -1 / 2 & 0
\end{array}\right]
$$

(iv) $M$ can be endowed with an integrable para-quaternionic structure.

For the proof of this theorem see [1, Section 9.1].

REMARK A. From (0.1) one can see that the sectional curvature of the plane $E_{1} \wedge E_{4}$ is vanishing and can easily verify that $M$ is a rank two symmetric space.

In the proof of Theorem 0.2 we use Wu's theory on symmetric holonomy systems. We denote by $H$ the 1-dimensional connected Lie subgroup of $G L(V)$, the Lie algebra of which is generated by endomorphism $m$. Wu has proved in [7], that every such $H$ can be realised as the holonomy group of a simply connected symmetric space $M$ whose tangent space at a point can be indentified with $V$ (in our case $V=\left(\mathbb{R}^{4}, g\right.$ ) of signature $(2,2))$ and with curvature tensor $R$. For Wu's construction one has to calculate the Lie algebra $\mathfrak{g}=\mathfrak{h} \oplus V$, (as a direct sum of vector spaces and not as the direct sum of Lie algebras) where the Lie brackets $[\cdot, \cdot]: \mathfrak{g} \wedge \mathfrak{g} \rightarrow \mathfrak{g}$ are defined by

$$
\begin{aligned}
{\left[h_{1}, h_{2}\right] } & =\left[h_{1}, h_{2}\right]_{\mathfrak{h}} \quad \text { if } \quad h_{1}, h_{2} \in \mathfrak{h}, \\
{\left[h_{1}, x\right] } & =h_{1}(x) \quad \text { if } \quad h_{1} \in \mathfrak{h}, x \in V, \\
{[x, y] } & =R(x, y) \quad \text { if } \quad x, y \in V .
\end{aligned}
$$


Then if we show the solvability of $G$, the corresponding Lie group of $\mathfrak{g}$, we know that the homogeneous manifold $M=G / H$ is diffeomorphic to a Euclidean space (in our case $\mathbb{R}^{4}$ ).

\section{Construction}

In this section using Theorem 0.2 we construct a pseudo-Riemannian manifold of signature $(2,2)$ on $\mathbb{R}^{4}$ such that this manifold satisfies the Osserman timelike condition with curvature tensor given by the formulas (0.1).

TheOREM 1.1. Let $M=\mathbb{R}^{4}$, let $\left(u_{1}, u_{2}, u_{3}, u_{4}\right)$ be the Cartesian coordinates and

$$
\begin{aligned}
g=\frac{1}{6}\left(v_{2}^{2} d v_{1} \otimes d v_{1}+\right. & \left.v_{1}^{2} d v_{2} \otimes d v_{2}-v_{1} v_{2}\left[d v_{1} \otimes d v_{2}+d v_{2} \otimes d v_{1}\right]\right) \\
& -\frac{1}{2}\left(\left[d v_{1} \otimes d v_{4}+d v_{4} \otimes d v_{1}+d v_{2} \otimes d v_{3}+d v_{3} \otimes d v_{2}\right]\right)
\end{aligned}
$$

Then $\left(\mathbb{R}^{4}, g\right)$ is a timelike Osserman rank two symmetric space.

PROOF: Let $\mathfrak{g}$ be the 5-dimensional Lie algebra defined by relations (0.1)-(0.3). If we change basis of $V$ and take the new basis $F=\left\{m, F_{1}, F_{2}, F_{3}, F_{4}\right\}$ where :

$$
F_{1}=\frac{\left(E_{1}+E_{4}\right)}{2}, \quad F_{2}=\frac{\left(E_{2}-E_{3}\right)}{2}, \quad F_{3}=\frac{\left(E_{2}+E_{3}\right)}{2}, \quad F_{4}=\frac{\left(E_{1}-E_{4}\right)}{2},
$$

then the only nonvanishing comutators in the Lie algebra $\mathfrak{g}$ are :

$$
\left[F_{1}, F_{2}\right]=m, \quad\left[m, F_{1}\right]=F_{3}, \quad \text { and } \quad\left[m, F_{2}\right]=-F_{4} .
$$

The algebra $\mathfrak{g}$ defined by formulas (0.3) and (1.3), is nilpotent because $\mathcal{D}^{4} \mathfrak{g}=\{0\}$ (see $[6])$, and so it is solvable. Now, the Campbell-Hausdorff series and the formulas (1.3) enables us to express the group multiplication in terms of coordinates. More precisely, let $X, Y$ be the elements of $\mathfrak{g}$, and let $X=\left(x_{i}\right)$, and $Y=\left(y_{i}\right), i=0, \cdots, 4$, be their coordinates in basis $F$. Then we have

$$
\begin{aligned}
Z & =X \cdot Y=Z(X, Y)=\left(z_{0}, z_{1}, \ldots, z_{4}\right), \text { where: } \\
z_{0} & =z_{0}(X, Y)=x_{0}+y_{0}+\frac{1}{2}\left(x_{1} y_{2}-x_{2} y_{1}\right), \\
z_{1} & =z_{1}(X, Y)=x_{1}+y_{1}, \quad z_{2}=z_{2}(X, Y)=x_{2}+y_{2}, \\
z_{3} & =z_{3}(X, Y)=x_{3}+y_{3}+\frac{1}{2}\left(x_{0} y_{1}-x_{1} y_{0}\right)+\frac{1}{12}\left(y_{1}-x_{1}\right)\left(x_{1} y_{2}-x_{2} y_{1}\right), \\
z_{4} & =z_{4}(X, Y)=x_{4}+y_{4}-\frac{1}{2}\left(x_{0} y_{2}-x_{2} y_{0}\right)-\frac{1}{12}\left(y_{2}-x_{2}\right)\left(x_{1} y_{2}-x_{2} y_{1}\right) .
\end{aligned}
$$


Since $\mathfrak{g}$ is nilpotent, the Campbell-Hausdorff formula defines a global diffeomorphism between $G$ and $\mathfrak{g}$. Let $M$ be the image of $V$ via the exponential mapping, so $M \cong$ $V$ is a homogeneous submanifold of $G$. Then we identify $M$ with $\mathbb{R}^{4}$, forgetting the first component, so $M=\left\{\left(x_{0}, x_{1}, x_{2}, x_{3}, x_{4}\right) \in \mathbb{R}^{5} \mid x_{0}=0\right\}$. For each $x \in G$, and $v \in M$, we consider the left translation $L_{x} v=x \cdot v$. In general, we get some element of $G$, even we take $x$ from $M$, because $V$ is not a subalgebra of $\mathfrak{g}$. Then we take the projection on $M$ in the direction of $\exp \mathfrak{h}$, and we consider the mapping $L_{x}^{h}=\pi_{M}^{h} \circ L_{x}: M \longmapsto M$. For geometrical reasons we know that this mapping is well defined, which means there exists a unique $h \in \exp h, \quad h=h(x, v)$, such that $\left(L_{x} v\right) \cdot h \in M$. Obviously, this map is a diffeomorphism since it is a composition of two diffeomorphisms. If $x=\left(x_{0}, x_{1}, x_{2}, x_{3}, x_{4}\right) \in G$ and $v=\left(0, v_{1}, v_{2}, v_{3}, v_{4}\right) \in M$ then $L_{x}^{h} v=\left(0, u_{1}, u_{2}, u_{3}, u_{4}\right) \cong\left(u_{1}, u_{2}, u_{3}, u_{4}\right)$, where

$$
\begin{aligned}
& u_{1}=x_{1}+v_{1}, \quad u_{2}=x_{2}+v_{2} \\
& u_{3}=x_{3}+v_{3}+x_{0} v_{1}+\frac{1}{2} x_{0} x_{1}+\frac{1}{6}\left(x_{1}+2 v_{1}\right)\left(x_{1} v_{2}-x_{2} v_{1}\right), \\
& u_{4}=x_{4}+v_{4}-x_{0} v_{2}-\frac{1}{2} x_{0} x_{2}-\frac{1}{6}\left(x_{2}+2 v_{2}\right)\left(x_{1} v_{2}-x_{2} v_{1}\right) .
\end{aligned}
$$

It still remains to calculate explicitly the metric on the manifold $M$. We know $\mathfrak{g}=T_{e} G=\mathfrak{h} \oplus V$, and $V \cong M$. But we changed the basis of $V$, and in our metric all of vectors from the basis $F_{V}=\left\{F_{i}, i=1, \ldots, 4\right\}$ are isotropic and

$$
\left\langle F_{1}, F_{4}\right\rangle=\left\langle F_{3}, F_{2}\right\rangle=-\frac{1}{2}, \quad \text { and } \quad\left\langle F_{i}, F_{j}\right\rangle=0 \text { otherwise. }
$$

Now if we take $X=\sum x_{i} F_{i}, Y=\sum y_{i} F_{i} \in V$ then, using the formulas (1.6), we get the metric on $T_{0} M$ in the coordinates: $g_{0}(X, Y)=-\left(x_{1} y_{4}+x_{2} y_{3}+x_{3} y_{2}+x_{4} y_{1}\right) / 2$.

To finish our construction we use the formula for the transport of the metric from $T_{0} M$ to $T_{v} M: g_{v}(X, Y)=g_{0}\left(\left(L_{v^{-1}}^{h}\right)_{*}(v) X,\left(L_{v^{-1}}^{h}\right)_{*}(v) Y\right)$. If $X=\left(x_{i}\right) \in G$, from relations (1.4) we find the coordinates of its group inverse $X^{-1}=\left(-x_{i}\right), i=0, \cdots, 4$. Now, we calculate $\left(L_{v^{-1}}^{h}\right)_{*}(v)$ from $(1.5)$ and then using the above formula for transporting the metric, we get the metric given by (1.1). We see from Remark $A$ that the manifold $M$ is of rank two.

REMARK B. By standard calculation of the curvature tensor from the metric on $M$ we get that the only nonzero component of the curvature tensor in the basis $F_{V}$ is $R_{1221}^{M_{F}}=1 / 2$. But the prescribed curvature tensor $R$, given by the components $(0.1)$, is calculated in basis $E_{V}$. One can easily find the connection between the bases $E_{V}$ and $F_{V}$ using (1.2), and after that verify that all components of the tensor $R^{M}$ are the same as those of the prescribed tensor $R$. 


\section{REFERENCES}

[1] N. Blažić, N. Bokan and Z. Rakić, 'Characterization of 4-dimensional Osserman pseudoRiemannian Manifolds', (preprint).

[2] Q.S. Chi, 'A curvature characterization of certain locally rank-one symmetric spaces', $J$. Differential Geom. 28 (1988), 187-202.

[3] Q.S. Chi, 'Curvature characterization and classification of rank-one symmetric spaces', Pacific. J. Math. 150 (1991), 31-42.

[4] P.B. Gilkey, A. Swann and L. Vanhecke, 'Isoparametric geodesic spheres and a conjecture of Osserman concerning the Jacobi operator', Quart. J. Math. Oxford. 46 (1995), 299-320.

[5] R. Osserman, 'Curvature in the eighties', Amer. Math. Monthly 97 (1990), 731-756.

[6] M.M. Postnikov, Lectures in geometry; Lie groups and Lie algebras, (English translation) (Mir Publishers, Moskva, 1986).

[7] H. Wu, 'Holonomy groups of indefinite metrics', Pacific. J. Math. 20 (1967), 351-392.

Faculty of Mathematics

University of Belgrade

Studentski Trg 16, P.P. 550

11000 Belgrade

Yugoslavia

e-mail: xpmfm28@yubgss21.bg.ac.yu 